\title{
Rendimiento de los deportistas con discapacidad intelectual enpruebas motrices. Diferencias en función de la edad y el género \\ Performance of the athletes with intellectual impairment in motor tests. Differences in age and gender
}

\author{
Jorge Abellán y Nieves María Sáez-Gallego \\ Universidad de Castilla-La Mancha
}

\begin{abstract}
Resumen. El primer objetivo de esta investigación fue examinar el rendimiento de deportistas con DI en 10 pruebas motrices, relacionadas con el empleo de las habilidades motrices. Adicionalmente, los resultados en todas las pruebas fueron correlacionados, tratando de establecer relaciones entre los diferentes rendimientos en cada una de ellas. El segundo objetivo fue explorar las diferencias en el rendimiento en función del sexo de los participantes. Y el tercer objetivo fue investigar las posibles diferencias en el rendimiento en las pruebas motrices en función de la edad de los participantes. Los resultados muestran una correlación muy alta entre la mayoría de los resultados en las pruebas motrices, indicando que los deportistas que obtenían puntuaciones altas lo lograban en gran variedad de pruebas. Además los hombres fueron significativamente mejores en las pruebas de tiro disco y desplazamiento utilizando diferentes apoyos. En cuanto al análisis en función de la edad, los deportistas del grupo juvenil fueron significativamente peores que el resto de sus compañeros en desplazamiento, precisión y raquetas y pelotas.

Palabras clave. discapacidad intelectual, pruebas motrices, deporte adaptado.
\end{abstract}

Abstract. The first aim of this paper was to examine the performance of athletes with ID in 10 motor tests, related with motor skills. Additionally, the results were correlated, trying to establish relationships between different performances in each of them. The second aim was to explore the differences in performance depending on the sex of the participants. The third aim was to investigate possible differences in motor performance tests based on the age of the participants. The results showed a high correlation between most of the results of the motor tests, indicating that athletes who were rated high they succeeded in a variety of tests. Men also were significantly better on tests of throwing disc and displacement using different props. In terms of age, athletes youth group were significantly worse than the rest of his teammates on the move, accuracy and racquets and balls.

Key words. intellectual impairment, motor test, adapted sport.

\section{Introducción}

La investigación en el deporte para personas con discapacidad está atrayendo el interés de los investigadores en ciencias del deporte, si bien las características de los participantes hacen que la consecución de objetivos de investigación sea más costosa. Según Pérez, Reina y Sanz (2012) estas dificultades se refieren fundamentalmente a: la escasez de las muestras obtenidas, con un número muy pequeño y características muy heterogéneas; la falta de test o pruebas estandarizadas; y la ausencia de coordinación multidisciplinar. Además, dentro de la propia discapacidad existen diferentes desarrollos, encontrándonos en la actualidad con el deporte para personas con discapacidad intelectual (en adelante DI) con menos desarrollo científico queel resto de discapacidades (físicas y sensoriales) (Abellán, 2013).

La DI está referida a las limitaciones significativas que posee un individuo en el plano cognitivo, intelectual y de conducta adaptativa, que aparecen antes de los 18 años (Verdugo, 2003; AAIDD, 2010). Las personas con DI suelen tener una competencia motriz baja (Molina \& Beltrán, 2007), entendida como la dificultad de superar un problema motriz concreto (Ruiz, 1995). Además, según Carbó-Carreté, Giné y Guaria-Olmos (2013), las personas con DI tienen necesidades de apoyo muy importantes a la hora de practicar actividad física. Por todo ello, en los casos de personas con DI con mayores impedimentos se sugiere la utilización de pruebas motrices (Abellán \& Sáez-Gallego, 2014), teniendo en cuenta la cantidad de personas con DI que participan en actividades y eventos deportivos (Robertson \& Emerson, 2010).

SegúnAbellán y Sáez-Gallego (2014), las pruebas motrices son una alternativa a los deportes normalizados para aquellas personas con DI que, por sus características, tienen problemas para tomar decisiones en contextos deportivos (con limitaciones de tiempo y espacio). En estas pruebas motrices se presentan tareas deportivas muy concretas, en las que el proceso de percepción y toma de decisiones, así como la continua interacción de los limitadores que guían el comportamiento motor (organismo, entorno y tarea) (Newell, 1986), están guiados hacía una única solución posible: la ejecución buscada (Abellán \& Sáez-Gallego, 2014).

En el presente trabajo ampliamos el trabajo realizado por Abellán

Fecha recepción: 30-05-14- Fecha envío revisores: 02-06-14- Fecha de aceptación: 04-11-14 Jorge Abellán

jorge.abellan@uclm.es
(2013), en el que se analizó el rendimiento de deportistas con DI en pruebas motrices relacionadas con el lanzamiento. Se analizó el rendimiento de los participantes en tres pruebas (lanzamiento de precisión, lanzamiento a portería y lanzamiento a canasta) y en la puntuación total. Los participantes fueron clasificados en función de su rendimiento en el lanzamiento a portería, creando dos grupos de análisis, exitosos y menos exitosos. Los resultados mostraron que los participantes pertenecientes al grupo de exitosos fueron significativamente mejores en la prueba de lanzamiento de precisión y en la suma total de las tres pruebas realizadas. También fueron significativamente mejores en la prueba de lanzamiento a portería, lo que era previsible debido a que el autor clasificó a los deportistas en dos grupos exitosos y menos exitosos en función de sus resultados en la prueba de lanzamiento a portería. Aunque no se obtuvieron diferencias significativas en los resultados en la prueba de lanzamiento a canasta, también se observóla tendencia que los deportistas pertenecientes al grupo de exitosos obtuvieron una media de puntos superior. En base a los resultados obtenidos, Abellán (2013) afirma que los deportistas más exitosos con DI presentaron un mayor desarrollo de la habilidad de lanzar y que la pueden demostrar en diferentes situaciones. Estos resultados están de acuerdo con estudios previos, en deportistas sin discapacidad, en concreto en jugadores de fútbol, en los que los participantes más exitosos y expertos obtenían mejores resultados en más de una faceta del juego (Williams, 2000).

Las investigaciones centradas en el estudio del comportamiento motor en personas con DI se centran fundamentalmente en tratar de encontrar las diferencias entre las personas con y sin DI. Por ejemplo, Van Biesen et al. (2010) compararon el resto al servicio en deportistas de tenis de mesa, con y sin DI. Los resultados revelaron que los deportistas sin DI cometían menos fallos al adaptar su comportamiento motor para restar diferentes tipos de saques (con diferentes velocidades). También los deportistas sin DI presentaron un tiempo de reacción más rápido que sus compañeros con DI, sin embargo, los estudios centrados en la comparación de diferentes niveles de comportamiento motor, exclusivamente centrados en los deportistas con DI, son más escasos.

Recientemente se han llevado a cabo investigaciones centradas en el impacto de la práctica deportiva en las personas con discapacidad intelectual (Hoewen, Van der Putten \& Vlaskamp, 2014; OggGroenendaal, Hermans \& Claessens, 2014; Baran et al., 2013). Por ejemplo, Houwen et al. (2014) realizaron una revisión sistemática del efecto de intervenciones para mejorar los aspectos motores, cognitivos 
y sociales de las personas con discapacidad intelectual gravemente afectadas (como es el caso de nuestra muestra de trabajo). Entre los resultados destaca la idea de que ninguno de los artículos analizados refleja un efecto negativo en la intervención para la mejora de la motricidad. Sin embargo, en la mayoría de los artículos utilizados en esta revisión se muestra un efecto positivo en la práctica deportiva, ya que se encuentran resultados positivos a nivel de mejora de las habilidades motrices básicas y en menor medida en las habilidades específicas o empleadas en actividades recreativas.

El primer objetivo de esta investigación es examinar el rendimiento de deportistas con DI en 10 pruebas motrices. Adicionalmente, se correlacionarán los resultados en todas las pruebas, tratando de establecer relaciones entre los diferentes rendimientos en cada una de ellas. El segundo objetivo es explorar las diferencias en el rendimiento en función del sexo de los participantes. Y el tercer objetivo es investigar las posibles diferencias en el rendimiento en las pruebas motrices en función de la edad de los participantes. Teniendo en cuenta los resultados de estudios previos, se prevé que las pruebas con utilización de habilidades motrices similares (por ejemplo todas las que incluyan lanzamientos) obtengan correlaciones positivas. También se espera que los hombres obtengan mejores resultados que las mujeres en el resultado de las pruebas motrices y que los deportistas más jóvenes (menores de edad) consigan mejores puntuaciones que sus compañeros con más edad.

\section{Método}

\section{Participantes}

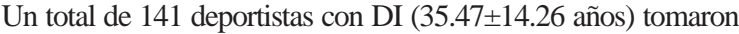
parte en el estudio. La muestra estuvo compuesta por 75 hombres (34.65 \pm 13.42 años) y 66 mujeres (36.39 \pm 15.20 años). Todos los participantes competían en el nivel G3 del deporte para personas con DI, cuyas características se explican a continuación:

Está dirigido a aquellos deportistas que por sus competencias motrices necesitan una adaptación rigurosa del reglamento o necesitan realizar pruebas motrices (individuales o colectivas) de la modalidad deportiva correspondiente adaptadas a sus posibilidades.

Las características de los deportistas participantes en este nivel son:

- Necesita ayuda verbal de un monitor o voluntario.

- Movimiento escaso y poca fuerza funcional en todas las extremidades.

- Dificultad significativa para comprender y llevar a cabo consignas verbales.

- Depende de ayudas técnicas para su movilidad(distintos tipos de sillas de ruedas, muletas, bipedestadores, andadores). (FECAM, 2012a: p.4)

Además, con el objetivo de analizar el rendimiento de los participantes en las pruebas motrices en función de la edad, se han creado tres diferentes grupos de análisis. El resultado de la distribución es el siguiente:

- Categoría juvenil, cuya edad es menor de 18 años (<18): 17 participantes (13.94 \pm 2.96 años)

- Categoría absoluta, cuya edad está comprendida entre los 18 y los 39 años (rango 18-39): 66 participantes (28.50 6.22 años)

- Categoría senior, compuesta por los participantes de 40 años en adelante (>40): 58 participantes (49.71 \pm 7.21 años)

Todos los participantes, sus tutores o padres (en el caso de estar inhabilitados o ser menores de edad) firmaron un documento de consentimiento informado para participar en los campeonatos regionales o nacionales de pruebas motrices de los que se extrajeron los datos utilizados en esta investigación. Además todos los participantes tenían la correspondiente licencia en vigor con su federación territorial correspondiente o en su defecto con Special Olympics España.

\section{Materiales}

Los materiales dependen de cada una de las diez pruebas motrices realizadas (para más detalles ver la sección procedimiento).Acontinua- ción se enumeran todos los materiales utilizados, divididos en pruebas y cuya distribución en la práctica puede observarse en la Figura 1.

Desplazamientos (3 aros de $60 \mathrm{~cm}$. de diámetro y 3 picas de $15 \mathrm{~cm}$. de altura); Tiro de disco (1 disco de fieltro y 1 portería de fútbol sala); Recepción de pelotas (1 pelota de plástico de $22 \mathrm{~cm}$. de diámetro); Lanzamiento a canasta (5 pelotas de gomaespuma de $22 \mathrm{~cm}$. de diámetro y 1 mini-canasta de baloncesto de $1 \mathrm{~m}$. de altura y $1 \mathrm{~m}$. de diámetro); Lanzamiento de precisión (3 círculos de diferentes diámetros, 40, 60 y $80 \mathrm{~cm}$. respectivamente, y 1 pelota de tenis); Raquetas y pelotas (1 pelota de foam de $16 \mathrm{~cm}$. de diámetro y 1 raqueta de tenis junior); Equilibrio dinámico (1 línea de plástico de $2 \mathrm{~m}$. de longitud y $10 \mathrm{~cm}$. de grosor, 1 plinto de $2 \mathrm{~m}$. de longitud y 1 banco sueco de $2 \mathrm{~m}$. de longitud); Desplazamiento con diferentes apoyos (6 picas con sus bases y 3 cintas para montar 2 obstáculos de 80, 60 y $40 \mathrm{~cm}$. respectivamente); Tiro a portería (1 portería de fútbol sala y 5 balones de gomaespuma de $22 \mathrm{~cm}$. de diámetro); Bowling (10 bolos y 1 bola de plástico de 500 gr. de peso).

\section{Procedimiento}

Todos los participantes completaron las siguientes diez pruebas motrices. Las variables dependientes analizadas se refieren al rendimiento de cada uno de los participantes en las pruebas motrices realizadas, la puntuación máxima que se puede obtener en cada una de las pruebas es de 10 puntos. En cada una de las pruebas, siempre que el deportista se presente en la prueba con la intención de realizarla conseguirá 1 punto y si no se presenta o lo hace con la intención de no realizarla recibirá 0 puntos. El desarrollo de las pruebas se explica a continuación (Abellán, 2013; Abellán \& Sáez-Gallego, 2014; FECAM, 2012b; SOE, 2010), además la Figura 1 muestra la representación gráfica sobre la disposición de los materiales en las distintas pruebas:

- Desplazamiento: los deportistas deben completar un circuito compuesto por 3 aros (que hay que pasar por encima), desplazarse entre 3 conos (haciendo zig-zag) y pasar por encima de 3 picas a una altura de $10-15 \mathrm{~cm}$. Sumará 1 punto siempre que pase algún obstáculo (aro, cono o pica)

- Tiro de disco: los deportistas deben realizar 5 lanzamientos con un disco de fieltro a una portería vacía desde 5 posiciones, que se distribuyen en $30^{\circ}$, tomadas desde el centro de la portería. Conseguirán 1 punto siempre que metan gol desde la posición 1 (en el centro de la portería) y 2 puntos siempre que metan gol desde las posiciones 2, 3, 4 y 5 .

Recepción de pelotas: el deportista debe tratar de atrapar un total de 7 lanzamientos de pelotas, realizados desde distancias de 1,2 y $3 \mathrm{~m}$. Obtendrá 1 punto siempre que atrape la pelota desde $1 \mathrm{~m}$. y $2 \mathrm{~m}$. y conseguirá 2 puntos siempre que atrape la pelota desde $3 \mathrm{~m}$.

- Lanzamiento a canasta: los deportistas deberán tratar de meter 5 pelotas desde 1,2 y $3 \mathrm{~m}$. a una canasta a $1 \mathrm{~m}$. de altura y $1 \mathrm{~m}$. de diámetro. Se valora con 1 punto si consigue anotar desde la distancia de $1 \mathrm{~m}$. y 2 puntos por cada canasta anotada desde 2 y $3 \mathrm{~m}$.

- Lanzamiento de precisión: los deportistas deben lanzar pelotas a 3 círculos de diámetros de 40,60 y $80 \mathrm{~cm}$. desde una distancia de $2 \mathrm{~m}$. El total de lanzamientos a realizar será de 7. El lanzamiento tendrá un valor diferente en función del tamaño del aro atravesado: 1 punto por el aro grande y mediano y dos puntos por el aro pequeño.

- Raquetas y pelotas: el deportista debe mantener una pelota encima de la raqueta y desplazarse $10 \mathrm{~m}$. sin que la pelota se caiga. Se le descontará 1 punto (de un total de 10) cada vez que se le caiga la pelota durante el recorrido.

- Equilibrio dinámico: los deportistas deben completar un circuito en el que se deben desplazar encima de una línea ( $2 \mathrm{~m}$. de longitud y 10 $\mathrm{cm}$. de grosor), después caminar encima de una superficie elevada (plinto) de $2 \mathrm{~m}$. y caminar encima de un banco sueco de $2 \mathrm{~m}$. de longitud. El deportista conseguirá 2 puntos si se desplaza con éxito por la línea marcada en el suelo, anotará 3 puntos si se desplaza con éxito encima del plinto y obtendrá 4 puntos si se desplaza con éxito por el banco sueco.

- Desplazamiento con diferentes apoyos: el deportista debe desplazarse en bipedestación pasando por debajo de una cinta situada a $1 \mathrm{~m}$. 
de altura, después debe pasar en cuadrupedia debajo de una cinta situada a $60 \mathrm{~cm}$. de altura, y finalmente debe reptar o rodar por debajo de una cinta situada a $40 \mathrm{~cm}$. de la base del cuerpo del deportista tendido en el suelo. Se anotarán 2 puntos si el deportista se desplaza con éxito bajo la altura de $1 \mathrm{~m}$., conseguirá 3 puntos si se desplaza con éxito bajo la altura de $60 \mathrm{~cm}$. y obtendrá 4 puntos si se desplaza con éxito bajo la altura de $40 \mathrm{~cm}$.

- Tiro a portería: los deportistas deberán golpear con el pie pelotas con el objetivo de introducirlas en una portería. Se realizarán 5 lanzamientos desde diferentes puntos, todos ellos a $3 \mathrm{~m}$. de distancia de la portería. Si el deportista anota el lanzamiento desde la zona 1 recibe 1 punto. Si anota lanzamientos desde las otras cuatro zonas (2, 3, 4 y 5) recibe 2 puntos.

- Bowling: los deportistas deben tratar de derribar 10 bolos, situados a una distancia de $3 \mathrm{~m}$. Dispondrán de 1 lanzamiento. Se anotará 1 punto por cada bolo derribado.

Desplazamiento
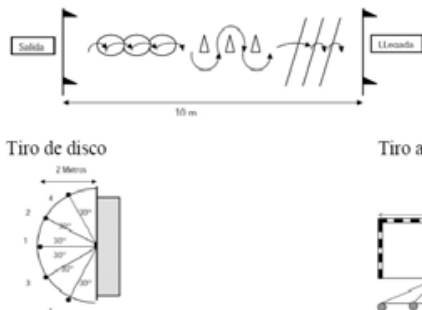

Recepción de pelotas

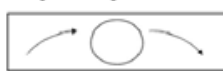

Tiro a porteria
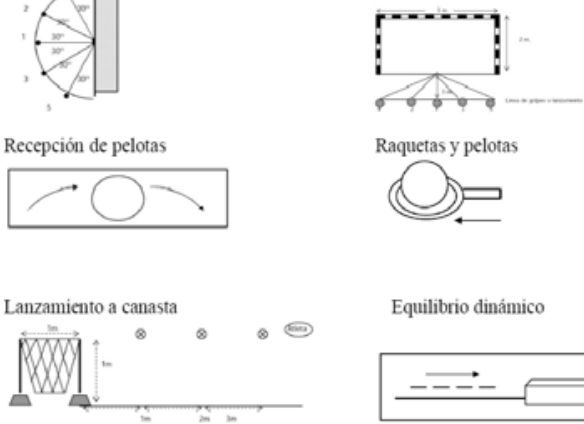

Equilibrio dinámico

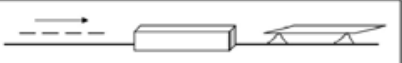

Lanzamiento de precisión

Desplazamiento con diferentes apoyos
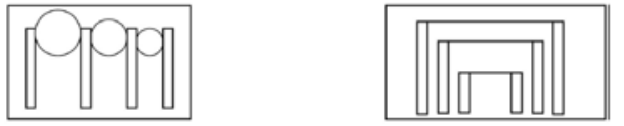

Bowling

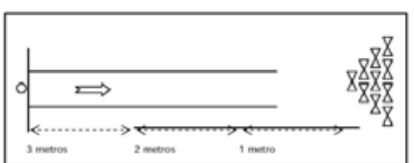

Figura 1. Desarrollo de las pruebas motrices (Abellán \& Sáez-Gallego, 2014; FECAM, 2012b).

Estadísticos descriptivos de las puntuacion es obtenidas en las pruebas motrices. (M $\pm D T)$

\begin{tabular}{lccccc}
\hline \multicolumn{5}{c}{ SEXO } & \multicolumn{3}{c}{ EDAD } \\
\hline \multicolumn{1}{c}{ PRUEBAS } & $\begin{array}{c}\text { Hombre } \\
(\mathrm{N}=75)\end{array}$ & $\begin{array}{c}\text { Mujer } \\
(\mathrm{N}=66)\end{array}$ & $\begin{array}{c}\text { Juvenil } \\
(\mathrm{N}=17)\end{array}$ & $\begin{array}{c}\text { Absoluta } \\
(\mathrm{N}=66)\end{array}$ & $\begin{array}{c}\text { Senior } \\
(\mathrm{N}=58)\end{array}$ \\
\hline Desplazami ento & $8.57 \pm 1.68$ & $8.91 \pm 1.79$ & $7.53 \pm 2.92$ & $9.02 \pm 1.41$ & $8.76 \pm 1.50$ \\
Tiro Di sco & $7.67 \pm 1.93$ & $6.44 \pm 2.83$ & $6.59 \pm 2.55$ & $7.38 \pm 2.17$ & $6.91 \pm 2.74$ \\
Recepción Pelotas & $8.75 \pm 1.51$ & $9.06 \pm 1.35$ & $8.76 \pm 1.35$ & $8.98 \pm 1.31$ & $8.83 \pm 1.61$ \\
Canasta & $5.29 \pm 2.39$ & $5.50 \pm 2.76$ & $4.29 \pm 2.59$ & $5.76 \pm 2.34$ & $5.29 \pm 2.74$ \\
Precisión & $6.91 \pm 2.16$ & $6.65 \pm 2.81$ & $4.76 \pm 2.51$ & $6.95 \pm 2.22$ & $7.19 \pm 2.84$ \\
Porteńa & $6.35 \pm 2.28$ & $6.67 \pm 2.24$ & $5.82 \pm 2.19$ & $6.38 \pm 2.36$ & $6.83 \pm 2.14$ \\
Raquetas Pelotas & $8.61 \pm 1.96$ & $8.14 \pm 2.75$ & $6.59 \pm 3.35$ & $8.42 \pm 2.01$ & $8.88 \pm 2.18$ \\
Equilibrio Dinámico & $7.76 \pm 2.29$ & $8.18 \pm 2.24$ & $7.94 \pm 2.72$ & $8.18 \pm 2.03$ & $7.71 \pm 2.39$ \\
Desplazami ento & & & & & \\
Apo yos & $8.93 \pm 2.19$ & $7.59 \pm 3.02$ & $8.76 \pm 2.75$ & $8.39 \pm 2.67$ & $8.07 \pm 2.77$ \\
Bowling & $3.45 \pm 2.89$ & $2.59 \pm 2.61$ & $3.41 \pm 2.71$ & $3.18 \pm 2.78$ & $2.79 \pm 2.83$ \\
\hline & & & & & \\
\hline
\end{tabular}

\section{Análisis estadístico}

Los datos provenientes del registro del rendimiento de todos los participantes en cada una de las pruebas motrices se vertieron a un documento de Excel y de ahí a SPSS 19.0 (IBM Stadistics) para su análisis. Se realizó una prueba ANOVA de un factor para cada variable por separado. Con el fin de explorar las diferencias se aplicó la prueba Bonferroni como post-hoc y correlaciones con la prueba de correlación de Pearson.

\section{Resultados}

El análisis de los resultados se ha dividido en tres apartados, correspondientes con los diferentes objetivos del estudio: análisis descriptivo y correlacional, análisis inferencial en función del sexo y análisis inferencial en función de la edad.

\section{Análisis descriptivo y correlacional}

La Tabla 1 muestra los valores medios obtenidos en cada una de las pruebas motrices. Se observa que el valor más elevado aparece en la prueba de recepción de pelotas, mientras el valor más bajo lo obtiene la prueba de bowling.

La Tabla 2 muestra los índices de correlación obtenidos entre los resultados de las diferentes pruebas motrices realizadas, los cuales resultaron en general significativos y positivos. Como excepciones aparecen las correlaciones significativas y negativas entre el desplazamiento con apoyos y el lanzamiento a portería y entre el lanzamiento de precisión y los bowling.

Tabla 2 .

Correlaciones entre los resultados en cada una de las pruebas motrices realizadas.

\begin{tabular}{|c|c|c|c|c|c|c|c|c|c|c|}
\hline & 1 & 2 & 3 & 4 & 5 & 6 & 7 & 8 & 9 & 10 \\
\hline 1. Desplazamiento & 1 & & & & & & & & & \\
\hline 2. Tiro Disco & $.340^{* *}$ & 1 & & & & & & & & \\
\hline 3. Recepción Pelotas & .143 & -.007 & 1 & & & & & & & \\
\hline 4. Canasta & $.353 * *$ & $.293 * *$ & $.280^{* *}$ & 1 & & & & & & \\
\hline 5. Precisión & $.346^{* *}$ & $.296 * *$ & .161 & $.454^{* *}$ & 1 & & & & & \\
\hline 6. Portería & $.343 * *$ & -.008 & .014 & $.226 * *$ & $.308^{* *}$ & 1 & & & & \\
\hline 7. Raquetas Pelotas & $.454 * *$ & $.340^{* *}$ & .098 & .130 & $.197^{*}$ & $.201^{*}$ & 1 & & & \\
\hline 8. Equilibrio Dinámico & $.426^{* *}$ & $.479 * *$ & .058 & $.289 * *$ & -.011 & -.006 & $.205^{*}$ & 1 & & \\
\hline $\begin{array}{l}\text { 9. Desplazamiento } \\
\text { Apoyos }\end{array}$ & . 126 & $.183^{*}$ & .079 & .026 & -.060 & $-.207^{*}$ & .109 & $.270^{* *}$ & 1 & \\
\hline 10. Bowling & .047 & $.243 * *$ & -.038 & -.136 & $-191 *$ & $-.241 * *$ & -.099 & $.356 * *$ & $.328 * *$ & 1 \\
\hline
\end{tabular}

** Correl ación significativa a nivel .01 (bilateral)

* Correl ación significativa a nivel 05 (bilateral)

\section{Análisis en función del sexo}

La Figura 2 muestra los resultados de las variables analizadas en función del sexo. La prueba ANOVA de un factor revela diferencias significativas en función del grupo para las variables tiro de disco $(\mathrm{F}(2,140)$ $=9.2 ; p=.003)$ y desplazamiento con apoyos $(\mathrm{F}(2,140)=9.29 ; p=.003)$.

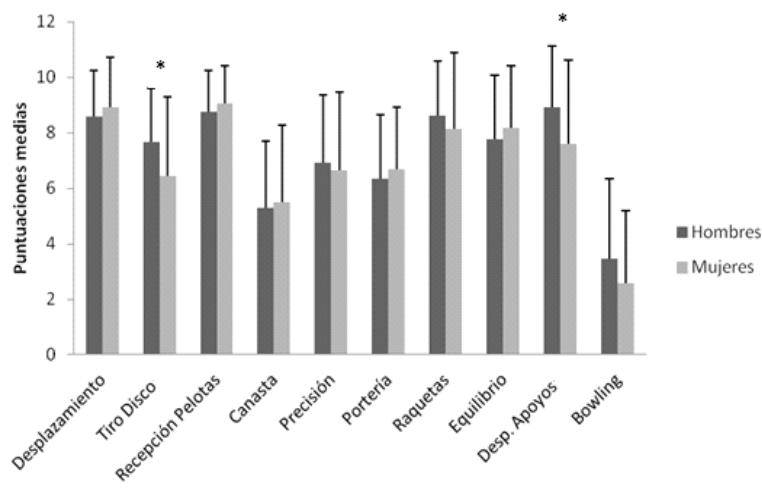

Figura 2. Punt uaciones medias de cada una de las pruebas motrices en función del género. El ast erisco $(*)$ indica di ferencias significativas para $p<.05$ 
En ambas variables, los hombres consiguen puntuaciones significativamente más altas que las mujeres.

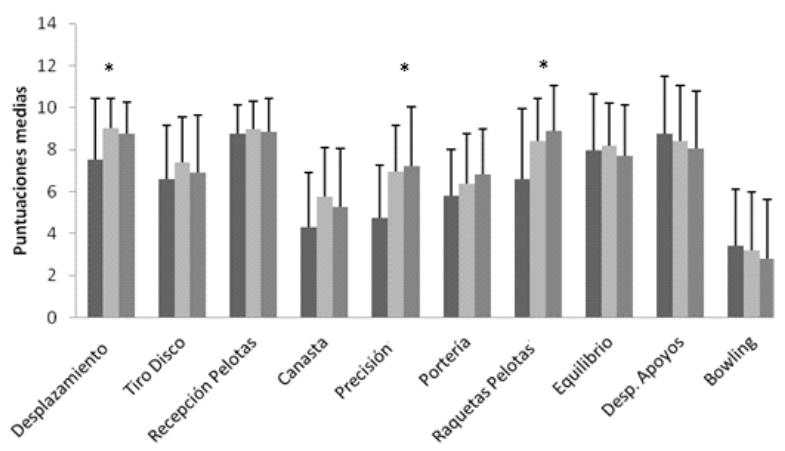

Figu ra 3. Punt uaciones medias de los grupos de edad en cada una de las pruebas motrices. El ast erisco (*) indica di ferencias signi ficativas para $p<.05$.

\section{Análisis en función de la edad}

La Figura 3 muestra los resultados en función de los tres grupos de edad creados. ANOVArevela diferencias significativas entre los grupos en las pruebas motrices de desplazamiento $(\mathrm{F}(2,140)=5.24 ; p=.006)$, precisión $(\mathrm{F}(2,140)=6.31 ; p=.002)$ y raquetas y pelotas $(\mathrm{F}(2,140)=$ 6.66; $p=.002$ ). Los resultados de la prueba de Bonferroni realizada como post-hoc, se exponen a continuación:

- Desplazamiento: el grupo juvenil obtiene significativamente menos puntuación que el grupo de categoría absoluta $(p=.004)$ y que el grupo senior $(p=.025)$

- Precisión: el grupo juvenil obtiene significativamente menos puntuación que el grupo de categoría absoluta $(p=.005)$ y que el grupo senior $(p=.002)$.

- Raquetas y pelotas: el grupo juvenil obtiene significativamente menos puntuación que el grupo de categoría absoluta $(p=.010)$ y que el grupo senior $(p=.001)$.

Por otra parte, los valores totales en cada grupo (Juvenil: $64.47 \pm$ DT; Absoluta: $72.65 \pm$ DT; Senior: 71.26 \pm DT) también revelan diferencias significativas $(\mathrm{F}(2,140)=3.61 ; p=.029)$, siendo el grupo juvenil el que se diferencia significativamente con el grupo de categoría absoluta en el análisis post-hoc ( $p=.024)$.

\section{Discusión}

En el presente trabajo se presenta una perspectiva de trabajo con el objetivo de investigar el deporte para personas con DI, mediante el empleo de pruebas motrices para que deportistas con DI con mayores impedimentos puedan participar en competiciones y mejorar sus resultados a través del entrenamiento. Por tanto, el primer objetivo de este artículo fue estudiar el rendimiento de los deportistas con DI en 10 pruebas motrices. Además, se han correlacionado los resultados de las pruebas tratando de buscar relaciones en el rendimiento obtenido en las diferentes pruebas. El segundo objetivo fue examinar las diferencias en función del sexo de los participantes. Finalmente el tercer objetivo fue investigar posibles diferencias en función de la edad de los participantes.

En referencia al primer objetivo, los resultados indican una fuerte correlación entre la mayor parte de las pruebas motrices, lo que indica que los deportistas que obtenían altas puntuaciones lo hacían en un buen número de pruebas. Estos resultados están de acuerdo con los previos obtenidos por Abellán (2013), en los que los deportistas más exitosos en las pruebas motrices relacionadas con el lanzamiento eran capaces de demostrarlo en diferentes situaciones de práctica.

Utilizando las agrupaciones de las pruebas motrices (Abellán \& Sáez-Gallego, 2014; SOE, 2010) podemos observar que los grupos de pruebas motrices de precisión (tiro disco, recepción de pelotas y raquetas y pelotas) y de lanzamientos (lanzamientos a canasta, lanzamiento de precisión y tiro a portería) presentan correlaciones muy altas. Por ejemplo, existe una alta correlación positiva entre los lanzamientos a canasta, los lanzamientos de precisión y los lanzamientos a portería (también en este caso con el equilibrio dinámico). O el caso de la recepción de pelotas que correlaciona positivamente con los lanzamientos a canasta. Podemos decir que el rendimiento de los deportistas, aun implicando habilidades diferentes, se muestra similar cuando se refiere a habilidades dentro de la familia de «manejo de objetos». De igual forma, las pruebas motrices de desplazamientos (desplazamientos, equilibrio dinámico y desplazamientos con diferentes apoyos) obtienen altas correlaciones. Por ejemplo, los resultados en la prueba de equilibrio dinámico correlacionan positivamente con la prueba de desplazamiento con diferentes apoyos. De acuerdo con Ureña, Ureña, Velandrino y Alarcón (2008) podemos decir que las pruebas que implican el control y manejo del propio cuerpo (desplazamientos, saltos y giros) obtienen resultados similares (grupo de pruebas motrices de desplazamientos), y también lo hacen aquellas pruebas relacionadas con el manejo de móviles (grupos de pruebas motrices de precisión y de lanzamientos). Sin embargo los resultados de la prueba de desplazamiento, que correlaciona positivamente con el tiro de disco, el lanzamiento a canasta, el lanzamiento de precisión, lanzamiento a portería, raquetas y pelotas y equilibrio dinámico, nos sugiere un cierto desarrollo global de la motricidad en los deportistas con DI.

Resultan contradictorios los resultados de la prueba de bowling, que además de ser los resultados más bajos de todas las pruebas motrices obtienen correlaciones negativas con pruebas relativamente similares, como son los lanzamientos de precisión y los lanzamientos a portería, y correlaciones positivas con la prueba de equilibrio dinámico. La posible explicación de estos resultados puede tener dos causas: en primer lugar, la puntuación media de esta prueba nos indica que estamos ante la prueba con más dificultad, en virtud de la baja puntuación media y la diferencia con el resultado del resto de pruebas, lo que supone la posibilidad de una variabilidad de resultados; en segundo lugar, las propias características de la muestra nos hace pensar en cierto grado de heterogeneidad en el desarrollo de las habilidades estudiadas, en esta prueba pero también en el resto de ellas, de acuerdo con Pérez et al. (2012), que se ha podido poner de manifiesto en el resultado de esta prueba en concreto. Sin embargo, se hace necesario una investigación más profunda en este sentido para obtener conclusiones más elaboradas. Por ejemplo sería interesante realizar estudios experimentales con la intención de mejorar las habilidades motrices de personas con DI, valorar el impacto de determinadas tareas de entrenamiento y explorar las diferencias en función del grado de discapacidad.

En cuanto al segundo objetivo propuesto, podemos decir que los hombres obtuvieron significativamente mejores resultados que las mujeres en las pruebas de tiro de disco y de desplazamiento con diferentes apoyos, aunque también obtienen mejores resultados, sin diferencias significativas, en el resto de pruebas evaluadas (excepto en la prueba de recepción de pelotas). Estos resultados nos sugieren que los hombres con DI tienen más desarrolladas las habilidades motrices evaluadas con estas pruebas motrices, además de una cierta superioridad física, referida a la mayor fuerza muscular. Las diferencias en función del género, a favor del masculino, están de acuerdo con estudios previos con escolares en la evaluación de diferentes habilidades motrices, como el atrape y el lanzamiento (Fernández-Losa, Cecchini \& Pallasá, 2013; Cecchini, Fernández-Losa, Pallasá \& Cecchini-Applegatte, 2012; Ruiz \& Graupera, 2003), además Bucco-dos Santos y Zubiaur-González (2013) encontraron superiores a los niños en equilibrio, esquema corporal y organización espacio-temporal, sin embargo las niñas fueron superiores en motricidad fina y motricidad gruesa.

$\mathrm{Al}$ analizar los resultados en función de la edad (tercer objetivo), encontramos que el grupo de edad juvenil obtiene significativamente peores resultados en las pruebas de desplazamiento, precisión, y raquetas y pelotas. Además, al analizar el resultado total de las pruebas motrices, se observa que el grupo de categoría absoluta es significativamente mejor que el grupo de categoría juvenil. Teniendo en cuenta que las limitaciones significativas que indican una discapacidad 
intelectual deben aparecen antes de los 18 años (Verdugo, 2003;AAIDD, 2010) podríamos pensar que los resultados del grupo juvenil podrían ser superiores al resto, sin embargo no es así. Los resultados aquí obtenidos están de acuerdo con los hallazgos de Cecchini etal. (2012) en su estudio con escolares. En dicho estudio los alumnos de mayor edad conseguían mejores resultados en pruebas relacionadas con el atrape. El hecho de que no existan diferencias entre la categoría absoluta y la categoría senior nos sugiere que los deportistas con DI son capaces de mantener un rendimiento relativamente estable durante mucho tiempo, debido sobre todo a la mínima exigencia física de las pruebas motrices evaluadas.

\section{Conclusiones}

A partir del estudio del rendimiento de deportistas con DI en 10 pruebas motrices y teniendo en cuenta los objetivos propuestos, podemos extraer las siguientes conclusiones:

- Existe una alta correlación positiva entre los rendimientos de las pruebas motrices que requieren el empleo de las mismas habilidades motrices, además de un desarrollo global de la motricidad de los deportistas con DI.

- Existen diferencias en el rendimiento en función del género, obteniendo los hombres mejores resultados que las mujeres, sin embargo estos resultados solo corresponden a las pruebas de tiro de disco y desplazamiento utilizando diferentes apoyos.

- Aparecen diferencias en función del género, los deportistas con DI mayores de 18 años son significativamente mejores que los menores.

\section{Agradecimientos}

Los autores agradecen a la Federación de Deportes para Personas con Discapacidad Intelectual de Castilla-La Mancha (FECAM) y a Special Olympics España (SOE) el acceso a los datos utilizados en este artículo.

\section{Referencias}

Abellán, J. (2013). Evaluación de las pruebas motrices relacionadas con el lanzamiento en deportistas con discapacidad intelectual. Retos. Nuevas tendencias en Educación Física, Deportey Recreación, 24, 63-66.

Abellán, J. \& Sáez-Gallego, N. M. (2014). Justificación de las pruebas motrices en el deporte para personas con discapacidad intelectual. Revista Iberoamericana de Psicología del Ejercicio y el Deporte, 9(1), 143-153.

Asociación americana de discapacidad intelectual y del desarrollo (AAIDD). (2010). Definition of intelectual disability. Recuperado de: http://aaidd.org/intellectual-disability/definition\#.VFN5obl0zIU (19/12/2012).

Baran, F., Aktop, A., Özer, D., Nalbant, S., Aglamis, E., Barak \& Hutzler, Y. (2013). The effects of a Special Olympics Unified Sports Soccer training program on anthropometry, physical fitness and skilled performance in Special Olympics soccer athletes and non-disabled partners. Research in Developmental Disabilities, 34(1), 695-709.

Bucco-dos Santos, L. \& Zubiaur-González, M. (2013). Desarrollo de las habilidades motoras fundamentales en función del sexo y del índice de masa corporal en escolares. Cuadernos de Psicología del Deporte, 13(2), 63-72.

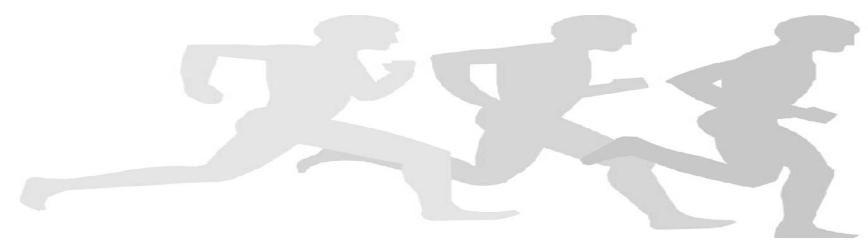

Carbó-Carreté, M., Giné, C. \& Guardia-Olmos, J. (2013). Estudio piloto para la elaboración de un cuestionario para la evaluación de hábitos y necesidades de apoyo para la actividad física en personas con discapacidad intelectual. Revista Iberoamericana de Psicología del Ejercicio y el Deporte, 8, 193-208.

Cecchini, J. A., Fernández-Losa, J. L., Pallasá, M. \& CecchiniApplegatte, C. (2012). El proceso de transferencia en el aprendizaje y desarrollo motor. Psicothema, 24(2), 205-210.

Federación de Deportes para Personas con Discapacidad Intelectual de Castilla-La Mancha (FECAM). (2012a). Batería pruebas motrices. Recuperado de: http://www.fecam.ws (10/06/2012).

Federación de Deportes para Personas con Discapacidad Intelectual de Castilla-La Mancha (FECAM). (2012b). Normativa 2012. Recuperado de: http://www.fecam.ws (16/06/2012).

Fernández-Losa, J., Cecchini, J.A. \& Pallasá, M. (2013). La recepción de balón en niños con edades comprendidas entre los 3 y los 12 años. Revista Internacional de Medicina y Ciencias de la Actividad Física y el Deporte, 50, 279-294.

Houwen, S., Van der Putten, A. \& Vlaskamp, C. (2014). A systematic review of the effects of motor interventions to improve motor, cognitive, and/or social functioning in people with severe or profound intellectual disabilities. Research in Developmental Disabilities, 35, 2093-2116.

Molina, J. P. \& Beltrán, V. J. (2007). Incompetencia motriz e ideología del rendimiento en educación física: el caso de un alumno con discapacidad intelectual. Motricidad. European Journal of Human Movement, 19, 165-190.

Ogg-Groenendaal, M., Hermans, H. \& Claessens, B. (2014). Asystematic review on the effect of exercise interventions on challenging behavior for people with intellectual disabilities. Research in Developmental Disabilities, 35, 1507-1517.

Pérez, J., Reina, R. \& Sanz, D. (2012). La Actividad Física Adaptada para personas con discapacidad en España: perspectivas científicas y de aplicación actual. Cultura, Ciencia y Deporte, 7, 213-224.

Robertson, J. \& Emerson, E. (2010). Participation in sports by people with Intellectual Disabilities in England: A brief report. Journal of Applied Research in Intellectual Disabilities, 23(6), 616-622.

Ruiz, L. M. (1995). Competencia motriz. Elementos para comprender el aprendizaje motor en educación física escolar. Madrid: Gymnos.

Ruiz, L. M. y Graupera, J. L. (2003). Competencia motriz y género entre escolares españoles. Revista Internacional de Medicinay Ciencias de la Actividad Física y el Deporte, 3(10), 101-111.

Special Olympics España (SOE), (2010). Batería de pruebas motrices. Special Olympics España. Recuperado de: http://specialolympics.es/ index.php?option=com_weblinks\&view=category\&id=38\&Itemid=569 (31/07/2012).

Ureña, N., Ureña, F., Velandrino, A. \& Alarcón, F. (2008). Estudio de la eficacia de un programa de intervención para la mejora de la habilidad de manejo de móviles en Primaria. Motricidad. European Journal of Human Movement, 21, 53-86.

Van Biesen, D., Vereller, J., Meyer, C., Mactavish, J., Van de Vliet, P. \& Vanlandewijck, Y. (2010). The ability of elite table tennis players with intelectual disabilities to adapt their service/return. Adapted Physical Activity Quarterly, 27, 242-257.

Verdugo, M.A. (2003). Análisis de la definición de discapacidad intelectual de la Asociación Americana sobre Retraso Mental de 2002. Siglo Cero: Revista Española sobre Discapacidad Intelectual,34(1), 5-19.

Williams, A. M. (2000). Perceptual skill in soccer: Implications for talent identification and development. Journal of Sport Sciences, 18, 737-750.

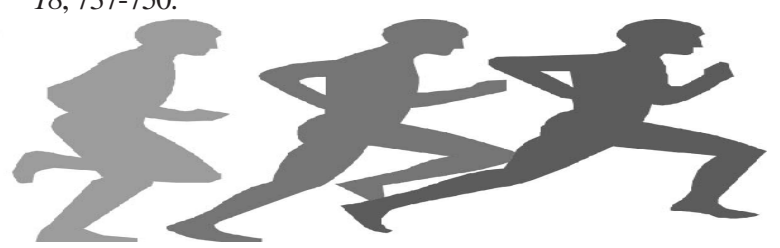

